02

\title{
Свободное вращение простых молекул и характеристические температуры в стеклообразных полимерах
}

\author{
(C) B.A. Рыжов \\ ФТИ им. А.Ф. Иофрфре РАН, \\ 194021 Санкт-Петербург, Россия \\ e-mail: v.ryzhov@mail.ioffe.ru
}

Поступила в редакцию 28.06.2021 г.

В окончательной редакции 16.08.2021 г.

Принята к публикации 31.08.2021 г.

Получены и проанализированы чисто вращательные спектры молекул $\mathrm{CO}$ и $\mathrm{HCl}$, добавленных в качестве зонда в полипропилен (ПП) и поливинилхлорид (ПВХ). Показано, что на форму полос этих спектров, отражающую степень возмущения вращательного движения, сильное влияние оказывает локальная динамика полимерной матрицы. По изменению интегральной интенсивности поглощения с температурой найдены температуры перехода от свободного вращения молекул $\mathrm{CO}$ и $\mathrm{HCl}$ к их либрации, а также температуры начала размораживания конформационной подвижности цепей макромолекул ПП и ПВХ.

Ключевые слова: вращательные ИК спектры, молекулярная динамика, полимеры, $\beta$-релаксация, стеклование.

DOI: $10.21883 /$ OS.2021.12.51738.2487-21

\section{Введение}

Автор посвящает статью профессору Михаилу Олеговичу Буланину, разработавшему новое направление молекулярной спектроскопии - трансляционную и вращательно-трансляционную спектроскопию простых молекул, методики которой $[1,2]$ в настоящей работе были использованы применительно к полимерам.

В то время как основными формами движения простых молекул являются колебания, а также поступательное и вращательное движение молекул как целого характерной, весьма важной особенностью макромолекул является внутримолекулярное движение в форме переориентаций атомных групп и поворотно-изомерных переходов в полимерных цепях. Именно оно определяет физико-химические и физико-механические свойства полимеров, а во многих случаях и кинетические особенности реакций с участием макромолекул.

Эти обстоятельства выдвигают изучение механизмов внутримолекулярного движения в ряд актуальных проблем физики полимеров. Следствием этого является все увеличивающееся число экспериментальных работ и стремление к более полному исследованию спектра молекулярной подвижности в широком диапазоне частот $10^{-2}-10^{13} \mathrm{~Hz}$ с привлечением методов диэлектрической спектроскопии, спектров рассеяния нейтронов и света, а также радиоспектроскопии люминесцентных и парамагнитных „меток“, вводимых в макромолекулы [3].

Используется в подобных исследованиях и спектроскопия среднего ИК диапазона, в частности метод конформационных зондов [4], когда в полимер вводится низкомолекулярное соединение, имеющее стабильные конформации, различимые по ИК спектру. Поскольку константа конформационного равновесия зависит от температуры, вид температурной зависимости соотношения интенсивностей конформационно-чувствительных полос позволяет определять температуру замораживания конформационных переходов зонда. Сравнивая объем, необходимый для конформационного перестроения зонда, с размерами подвижного элемента свободного объема матрицы, можно судить о том, движение какого фрагмента макромолекулы замораживается при данной температуре.

В представленной работе для изучения молекулярной динамики и межмолекулярных взаимодействий в полимерах применяется спектроскопия в дальнем ИК диапазоне $\left(10-300 \mathrm{~cm}^{-1}\right)$ и предложен метод, состоящий в использовании свободного вращения малых молекул, растворенных в полимере, позволяющий по степени заторможенности вращательного движения молекулы при ее переходе из газовой фазы в матрицу полимера получать информацию о релаксационной подвижности конкретных кинетических единиц самого полимера.

\section{Методика}

ИК спектры регистрировали на двух спектрометрах: в области от 10 до $100 \mathrm{~cm}^{-1}$ на приборе, разработанном в Ленинградском государственном университете и модернизированном в ФТИ с помощью приемника ОАП-7 и новой системы фильтрации, и от 100 до $300 \mathrm{~cm}^{-1}$ на спектрометре „Hitachi“ FIS-21 (Япония). Разрешение при отношении сигнала к шуму порядка 100 составляло $1-2 \mathrm{~cm}^{-1}$. Точность определения частоты максимума полосы составляла $1-2 \mathrm{~cm}^{-1}$. Погрешность измерения коэффициента поглощения $k(v)$, включая 
экспериментальные погрешности, была не более $10 \%$. Интегральная интенсивность зарегистрированных полос поглощения $A(T)$ определялась измерением площадей между спектральной кривой и базовой линией. Оно выполнялось при цифровой обработке экспериментальных данных с погрешностью, не превышающей $1 \%$. Объектами исследования служили полипропилен (ПП) и поливинилхлорид (ПВХ) с молекулярными массами в диапазоне $(1-2) \cdot 10^{5}$. Их пластины толщиной 400 и $200 \mu \mathrm{m}$ соответственно размещали в двух газовых кюветах с оптической длиной пути $10 \mathrm{~cm}$ и окнами из поли-4метилпентена-1: внутри рабочей кюветы и снаружи кюветы сравнения для компенсации поглощения самих полимеров и неадсорбированного газа. Кюветы заполнялись очищенной от паров воды моноокисью углерода $(\mathrm{CO})$ в опытах с ПП и хлористым водородом $(\mathrm{HCl})$ в случае ПВХ до давлений 350-700 mm $\mathrm{Hg}$. Число находящихся в контакте с газами поверхностей, через которые проходил световой пучок, было равно четырем.

Для достижения равновесия между газовой и растворенной фазой заполненная рабочая кювета выдерживалась перед съемкой около суток. Кювета сравнения также была заполнена газом, потому что, несмотря на небольшую длину пути кювет, в зарегистрированном нескомпенсированном спектре кроме полосы поглощения растворенного в полимере газа присутствовали и линии вращательного спектра газообразных $\mathrm{CO}$ и $\mathrm{HCl}$.

При изменении давления газа интенсивность полосы менялась линейно, что указывает на отсутствие насыщения в использованном интервале давлений.

Регулировка температуры при измерении спектров в температурном интервале от комнатной до $90 \mathrm{~K}$ осуществлялась с точностью $\pm 2^{\circ}$ изменением скорости потока холодного азота в теплообменнике, собранном на корпусе кюветы.

\section{Результаты и их обсуждение}

Приведенные к атмосферному давлению спектры СО и $\mathrm{HCl}$, растворенных в полимере, для одной поверхности, выраженные как $k(v)=\left(n p^{-1}\right) \ln \left(I_{0} / I\right)$ (где $n-$ число поверхностей, $p$ - давление в атмосферах, а $I_{0}$ и $I$ - пропускание кювет сравнения и рабочей) представлены на рис. 1 и рис. 2 соответственно.

Здесь же в относительных единицах оптической плотности для сравнения представлены чисто вращательные спектры поглощения молекул $\mathrm{CO}$ и $\mathrm{HCl}$ в газовой фазе.

Видно, что частоты разрешенной структуры ДИК спектров $\mathrm{CO}$ и $\mathrm{HCl}$, растворенных в полимере, совпадают с частотами линий вращательного спектра этих молекул в газовой фазе, а сама зарегистрированная полоса по форме и расположению напоминает вращательную ветвь с сильно размытой структурой. Эти остатки вращательной структуры в спектрах доказывают сохранение квантованного вращения части молекул СО

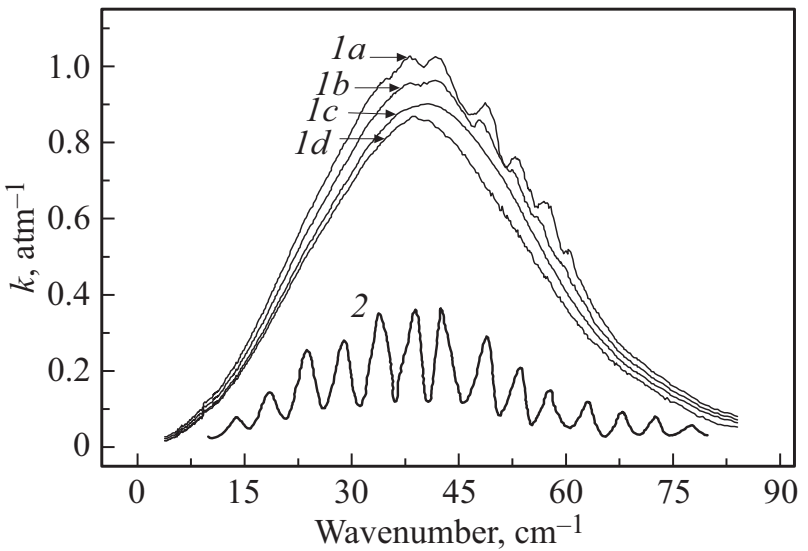

Рис. 1. Спектр погллощения моноокида углерода (СО). 1 - растворенной в ПП при $293(1 a), 260(1 b), 240$ (1c) и $200 \mathrm{~K}(1 d) ; 2$ - в газовой фазе при $293 \mathrm{~K}$ (в отн.ед. оптической плотности).

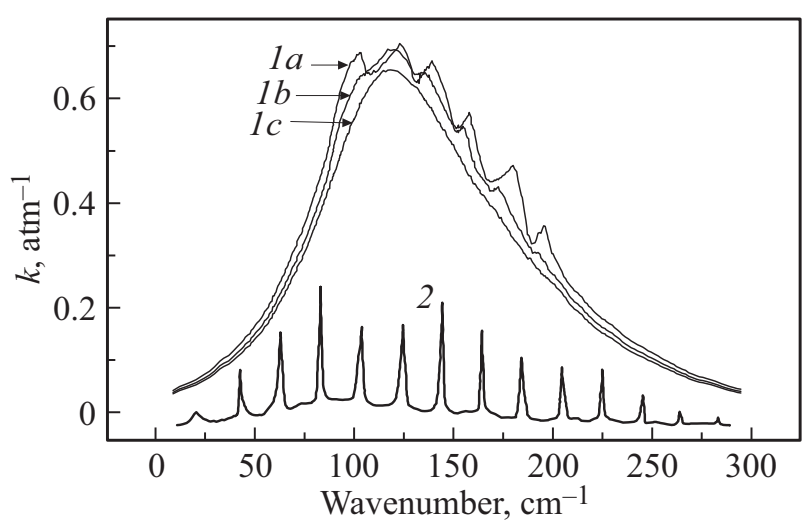

Рис. 2. Спектр поглощения хлористого водорода (HCl). 1 растворенной в ПВХ при 293 (1a), $220(1 b)$ и $185 \mathrm{~K}(1 c) ; 2-$ в газовой фазе при $293 \mathrm{~K}$ (в отн. ед. оптической плотности).

и $\mathrm{HCl}$ при переходе из газовой фазы в полимерную матрицу и подтверждают вращательно-либрационное происхождение представленных на рис. 1 и 2 полос. Значительная интенсивность этих полос указывает на большое количество внедрившихся в полимер молекул газа. Оценки, основанные на расчете интегральной интенсивности вращательного спектра, показывают, что одна поверхность полимера содержит несколько сотен молекулярных слоев с плотной упаковкой [2], т.е. газы действительно растворяются в полимерных пластинках, а не адсорбируются на их поверхности.

Полученные данные указывают на малую величину возмущения вращательного движения молекул $\mathrm{CO}$ и $\mathrm{HCl}$ в изученных полимерах при комнатной температуре. C понижением температуры контуры обнаруженных в настоящей работе полос претерпевают существенные изменения, обусловленные не только изменением межмолекулярных взаимодействий, но и молекулярной динамики молекул газа и макромолекул матрицы. Размы- 
вается тонкая структура полосы, свидетельствующая о постепенном замедлении и, наконец, полном прекращении вращательного движения растворенных в полимере молекул газа при температурах ниже температуры стеклования $T_{g} \approx 240 \mathrm{~K}$ для СО и $T_{g} \approx 190 \mathrm{~K}$ для $\mathrm{HCl}$. При этих температурах существенно суженная полоса характеризует лишь ограниченные крутильные колебания (либрацию) растворенных в полимере молекул.

Графики рис. 3 и 4 показывают, как изменяется интегральная интенсивность полос с температурой. Видно, что зависимость $A(T)$ не является монотонной, но свидетельствует о перестройках в системе „газ + полимер“ при определенных, связанных с релаксационными явлениями характеристических температурах. Действительно, метод широкодиапазонной $\left(10^{-3}-10^{12} \mathrm{~Hz}\right)$ диэлектрической спектроскопии $[3,5]$ и другие спектроскопические методы (ДМС, ЯМР, ЭСХА) [6,7] фиксируют релаксационные переходы при 30, 120, 190 и $270 \mathrm{~K}$ в ПП и при $20-30,145-170,190-210$ и $\sim 270$ К в ПВХ.

Для идентификации наблюдаемых переходов в нашем случае следует соотнести активационный объем растворенной молекулы (зонда) со свободным активационным объемом кинетической единицы, замороженной при температуре перехода. Последний при $T<T_{g}$ является суммой свободного объема, обусловленного неравновесной структурой стеклообразной полимерной матрицы, и свободного объема, который формируется локальной подвижностью различных атомных групп и фрагментов полимерных цепей и, значит, определяется размерами этих фрагментов. Когда в охлаждаемой системе свободный объем (и размер) какого-либо фрагмента макромолекулы становится соизмеримым со свободным объемом (и размером) зонда, его подвижность оказывается ограниченной, приводя к температурноиндуцированным изменениям спектральных параметров полосы поглощения. Таким образом, зная объем зонда (здесь порядка $20 \AA$ ), можно определить молекулярную группу или фрагмент цепи, которые участвовали в релаксационном переходе при данной температуре.

График зависимости $A(T)$ (рис. 4) фиксирует 3 таких перехода в ПВХ. Один из них при стекловании $\left(T_{g}=270 \mathrm{~K}\right)$, другой при $220 \mathrm{~K}$ очевидно связан с локальной конформационной подвижностью цепей этого полимера: локальным $\beta$-переходом в местах менее плотной упаковки при $T_{\beta}$. Такой переход состоит в поворотном движении звеньев корреляционного участка цепи (статистического сегмента) при участии однобарьерного $T \leftrightarrow G$-перехода [8]. И, несмотря на то, что свободный объем нанополости, необходимой для $\beta$-релаксации $\left(v_{\beta} \approx 1 \mathrm{~nm}^{3}\right)$ со статсегментом в качестве кинетической единицы движения, значительно превосходит ван-дервальсов объем зонда в системе „ПВХ $+\mathrm{HCl}^{“}$, тем не менее ее уменьшение при $T_{\beta}$ заметно (рис. 2) затормаживает вращение молекул, растворенных в полимере.

В этой чувствительности вращательных спектров к влиянию межмолекулярных сил, определяемых электрическими и оптическими параметрами взаимодей-

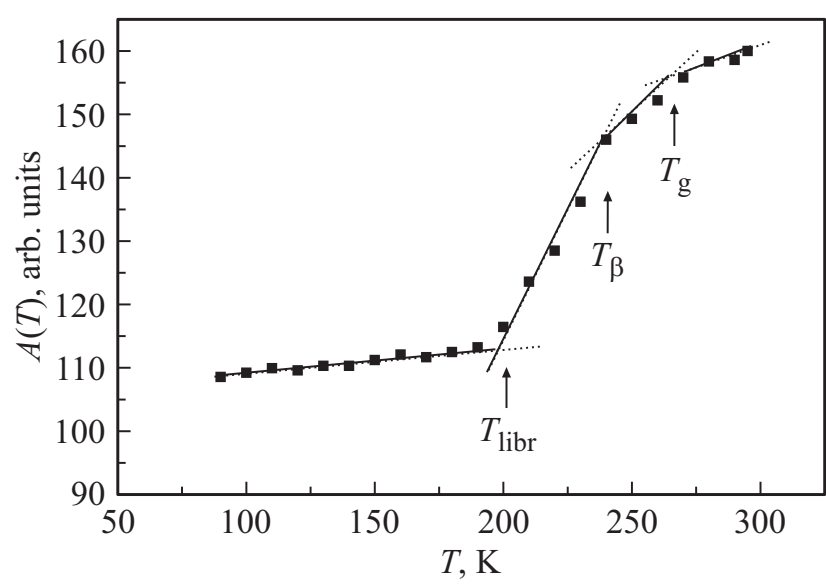

Рис. 3. Температурная зависимость интегральной интенсивности вращательно-либрационной полосы поглощения в длинноволновом ИК спектре $\mathrm{CO}$, растворенного в ПП. Стрелки отмечают положение температур стеклования $\left(T_{g}\right), \beta$-перехода $\left(T_{\beta}\right)$ и перехода к либрации $\left(T_{\mathrm{libr}}\right)$.

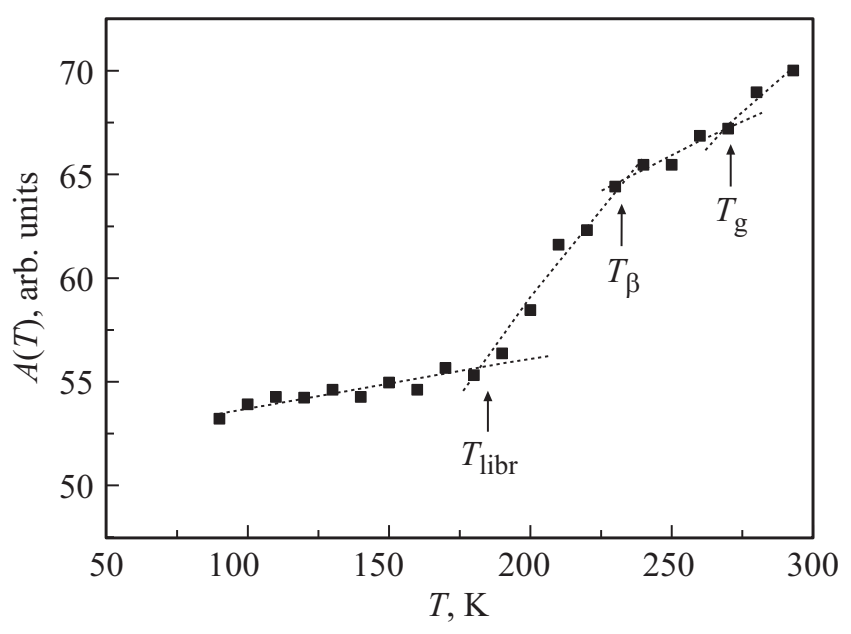

Рис. 4. Температурная зависимость интегральной интенсивности вращательно-либрационной полосы поглощения в длинноволновом ИК спектре $\mathrm{HCl}$, растворенного в ПВХ. Стрелки отмечают положение температур стеклования $\left(T_{g}\right), \beta$-перехода $\left(T_{\beta}\right)$ и перехода к либрации $\left(T_{\mathrm{libr}}\right)$.

ствующих молекул, и заключается главное преимущество предложенной в настоящей работе методики над методом колебательно-конформационных зондов. Газоподобное вращательное состояние молекулы (угловая скорость, продолжительность вращения и заселенность ее вращательного уровня) определяется не только размерами $\left(r_{\text {eff }}\right)$ молекулы, но также моментом инерции $\left(I=M r_{\text {eff }}^{2}\right)$ и спецификой межмолекулярного взаимодействия, определяющего высоту барьера для вращения,

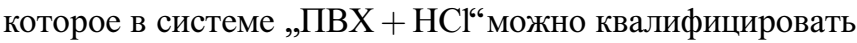
как водородную связь [9].

Третий переход в системе „ПВХ $+\mathrm{HCl}^{\text {“при }}$ $T_{\mathrm{libr}} \approx 190 \mathrm{~K}$, начиная с которой полоса поглощения полностью утрачивает свою дискретную структуру, без- 
условно вызван тем, что при этой температуре кинетическая энергия вращения молекул $\mathrm{HCl}\left(k T=127 \mathrm{~cm}^{-1}\right)$ становится сравнимой с потенциальным барьером, препятствующим ее вращению $\left(W_{0}=120 \mathrm{~cm}^{-1}[10]\right)$. И теперь из инерциального оно сводится главным образом к ограниченным качаниям (либрации) внутри квазистационарных потенциальных минимумов.

Поскольку тормозящий потенциал имеет ориентационную природу, т. е. зависит от ориентации молекул зонда по отношению к „стенкам“ матрицы, можно предположить, что переориентационное движение мономерных звеньев ПВХ к этому моменту еще не заморожено. Такие перескоки через барьер, очевидно, прекратятся при температуре $\delta$-перехода в ПВХ $\left(T_{\delta} \sim 20-30 \mathrm{~K}[6]\right)$, которая, к сожалению, лежит за пределами использованного температурного интервала.

Для другой изученной системы „ПП + $\mathrm{CO}^{\text {“ }}$ зависимость $A(T)$ (рис. 3) нарушает свой монотонный ход при 260, 240 и $200 \mathrm{~K}$. Поскольку, как и в предыдущем случае, макромолекулы матрицы в этой системе не имеют крупных боковых заместителей в основной цепи, a приписываемые подвижности монозвеньев и $\mathrm{CH}_{3}$ групп ПП, имеющих ван-дер-ваальсовые объемы, близки к таковым молекул аммиака, $\delta$-релаксация проявляется лишь при $T_{\delta} \approx 30 \mathrm{~K}$, и можно полагать, что найденные характеристические температуры отражают релаксационные процессы, связанные с подвижностью одного или нескольких фрагментов (сегментов) основной цепи. Так, излом при $T=260 \mathrm{~K}$ может быть связан со стеклованием ПП $\left(T_{g} \approx-13^{\circ} \mathrm{C}\right)$, т. е. с $\alpha$-переходом, состоящим в межмолекулярно-кооперированном движении соседних кинетических единиц (сегментов), имеющих, согласно представлениям, развитым в [8], длину, близкую к статсегменту макромолекул ПП.

Как видно из рис. 1, молекулы оксида углерода в этих условиях еще сохраняют свое свободное вращение. Однако, начиная с температур, близких к $T \approx 240 \mathrm{~K}$, вращательная структура ИК спектра системы „ПП + СО“ заметно размывается; происходит прогрессирующее исчезновение линий, отвечающих переходам с самых нижних вращательных уровней. Возможное объяснение наблюдаемых в спектрах изменений может быть связано с конденсацией молекул оксида $\left(T_{\text {кр }} \sim-34^{\circ} \mathrm{C}\right)$, сопровождающейся переходом от незначительно возмущенного инерциального вращения молекул СО к их переориентации при помощи беспорядочных поворотов на небольшие углы (вращательная диффузия по Френкелю [11]). Дальнейшее понижение температуры приводит к заметному упрощению формы контура полосы поглощения. При $T \approx 200 \mathrm{~K}$ ее параметры уже полностью соответствуют параметрам либрационной полосы в спектре жидкого оксида углерода, контур которой на значительном протяжении удовлетворительно описывается дисперсионной кривой [12]. Такие изменения могут быть истолкованы как следствие смены вращательной диффузии ограниченными крутильными колебаниями молекул $\mathrm{CO}$, растворенных в ПП. Вызваны они тем, что именно при
$T_{\beta}=200 \mathrm{~K}$ прекращаются конформационные перестроения в полимерной матрице, т.е. подавляется квазинезависимое сегментальное движение макромолекул по механизму $\beta$-релаксации [8]. Иными словами, замораживается свободный объем, обеспечивающий поворотноизомерные переходы в макромолекулах за счет скручивания корреляционного участка (сегмента) цепи и/или накопления в нем крутильно-колебательных смещений. Ниже температуры $T_{\beta}$ сегментальная конформационная подвижность в цепях полностью исчезает: сохраняются только малоамплитудные мелкомасштабные процессы, подобные ожидаемой в ПП при $30 \mathrm{~K} \delta$-релаксации. Возможность конкретизации механизма этой релаксации в настоящей работе ограничена использованием жидкого азота в качестве хладагента, но можно ожидать еще одного резкого (за счет крыльев) уменьшения интегральной интенсивности полосы либрационного поглощения. В условиях, когда свободный объем, связанный с локальной подвижностью монозвена ПП, станет сравним с вандер-ваальсовым объемом молекулы СО, ее подвижность ограничится лишь пределами потенциальной ямы, сформированной ближайшим окружением.

\section{Заключение}

В настоящей работе измерены и проанализированы длинноволновые ИК спектры молекул $\mathrm{CO}$ и $\mathrm{HCl}$, добавленных в качестве зонда в ПП и ПВХ. Спектры имеют вид аномально широкой асимметричной полосы поглощения с положением максимума при $\sim 40$ и $125 \mathrm{~cm}^{-1}$ для систем „ПП $+\mathrm{CO}^{\text {“ } ~ и ~ „ П В Х ~}+\mathrm{HCl}^{\text {“ } ~ с о о т в е т с т в е н н о . ~}$ Форма полос этих спектров отражает степень возмущения вращательного движения молекул $\mathrm{CO}$ и $\mathrm{HCl}$ при переходе от свободного вращения к вращательным колебаниям (либрации). По изменению интегральной интенсивности поглощения с температурой найдены температуры таких переходов.

Использованный метод изучения вращательного движения молекул, растворенных в полимерной матрице, позволяет, кроме того, установить природу релаксационных перестроений и конкретизировать на молекулярном уровне их механизмы. Такой подход представляет также интерес в теоретическом и прикладном плане. В теоретическом плане - так как, насколько нам известно, вращательное движение молекул газа в полимерах практически не изучено, а в прикладном - как метод изучения адсорбционных свойств и газопроницаемости полимерных материалов.

\section{Конфликт интересов}

Автор заявляет, что у него нет конфликта интересов. 


\section{Список литературы}

[1] Буланин М.О. Индуцированные спектры поглощения атомов и молекул. Труды ГОИ, 1981. Т. 46. В. 180. С. 1.

[2] Григорович Н.М., Тонков М.В. // Опт. и спектр. 1982. Т. 53. № 2. C. 194.

[3] Lunkenheimer P., Loidl A. Broadband Dielectric Spectroscopy / Ed. by Kremer F., Schonhals A. Berlin: Springer, 2002. P. 739.

[4] Ремизов А.Б., Камалова Д.И. // Высокомол. соед. 2007. T. 49. № 5. C. 779 .

[5] Рыжмов В.A. // Опт. и спектр. 2019. Т. 127. № 6. С. 895; Ryzhov V.A. // Opt. Spectrosc. 2019. V. 127. N 6. P. 979. doi 10.1134/S0030400X1912021X

[6] Sauer O. // J. Polym. Sci.: Polym. Symp. 1971. V. 2. P. 69.

[7] Iring K.J. (ed.). Structural Studies of Macromolecules by Spectroscopic Methods. London, U.K.: Wiely, 2001. P. 342.

[8] Берштейн В.А., Егоров В.М. Дифференциальная сканирующая калориметрия в физикохимии полимеров. Л.: Химия, 1990. 255 с.; Bershtein V.A., Egorov V.M. Differential Scanning Calorimetry of Polymers. Chichester: Ellis Horwood, 1992. 255 p.

[9] Chen J., Nie1 X.A., Jiang J.C., Zhou J.C. IOP Conf. Series: Materials Science and Engineering, 2017. V. 292. P. 012008.

[10] Буланин М.О., Орлова Н.Д. Спектроскопия взаимодействующих молекул / Под ред. Буланина М.О. Л.: ЛГУ, 1970. C. 84.

[11] Френкель Я.И. Кинетическая теория жидкостей. М., Л.: Изд. АН СССР, 1959. 463 с.

[12] Herrebout W.A., Van der Veken B.J., Medina A., Calvo Hernández A., Bulanin M.O. // Molec. Phys. 1999. V. 96. P. 1115. doi 10.1080/00268979909483054 